Received 15th May 2020, Accepted 17th August 2020

\section{Micro cell vesicle technology (mCVT): a novel hybrid system of gene delivery for hard-to- transfect (HTT) cells†}

\author{
Yi-Hsuan Ou, $\ddagger^{\text {a }}$ Shui Zou, $\ddagger^{\mathrm{a}}$ Wei Jiang Goh, ${ }^{\mathrm{a}, \mathrm{b}}$ Suet Yen Chong, ${ }^{\mathrm{c}, \mathrm{d}}$ \\ Gopalakrishnan Venkatesan, ${ }^{a}$ Matthias G. Wacker, ${ }^{a}$ Gerrit Storm, ${ }^{c}$ \\ Jiong-Wei Wang, (iD ${ }^{* c, d, e}$ Bertrand Czarny, ${ }^{* f}$ Giorgia Pastorin (iD) *a,b and \\ Esther C. Y. Woon (D)*a,g
}

A hybrid gene delivery platform, micro Cell Vesicle Technology (mCVT), produced from the fusion of plasma membranes and cationic lipids, is presently used to improve the transfection efficiency of hard-to-transfect (HTT) cells. The plasma membrane components of mCVTs impart specificity in cellular uptake and reduce cytotoxicity in the transfection process, while the cationic lipids complex with the genetic material and provide structural integrity to mCVTs.

\section{Introduction}

Gene delivery is the process of introducing foreign DNA into eukaryotic cells, such as the delivery of therapeutic or functional genes for alterations of cellular processes in both basic research (e.g. in drug discovery and in mechanistic studies of functional proteins ${ }^{1}$ ) and clinical applications (e.g. gene therapy). Effective gene transfection requires not only the successful introduction of foreign nucleic acids into the cells but also their subsequent trafficking in and expression by the cells. This is a challenging task as DNA molecules are inher-

\footnotetext{
${ }^{a}$ Department of Pharmacy, National University of Singapore, Singapore.

E-mail:phapg@nus.edu.sg,esther.woon@nus.edu.sg

${ }^{b}$ NUS Graduate School for Integrative Sciences and Engineering, Centre for Life Sciences (CeLS), Singapore

'Department of Surgery, Yong Loo Lin School of Medicine, National University of Singapore, Singapore.E-mail: surwang@nus.edu.sg

${ }^{d}$ Cardiovascular Research Institute (CVRI), National University Heart Centre Singapore (NUHCS) and National University Health System (NUHS), Singapore ${ }^{e}$ Department of Physiology, Yong Loo Lin School of Medicine, National University of Singapore, Singapore

${ }^{f}$ School of Materials, Science and Engineering \& Lee Kong Chian School of Medicine (LKCmedicine), Nanyang Technological University, 50 Nanyang Avenue, Singapore 639798.E-mail: bczarny@ntu.edu.sg

${ }^{8}$ School of Pharmacy, University College London, 29-39 Brunswick Square, London WCIN IAX, UK

† Electronic supplementary information (ESI) available. See DOI: 10.1039/ d0nr03784b

‡ Co-first authors, authors contributed equally to this paper.
}

ently anionic, thus poorly internalized by cells in their native 20 form. ${ }^{2}$ There is therefore tremendous interest in the development of effective gene delivery systems. Over the last decades, a great variety of carrier systems has been explored, ranging from virus-based delivery systems to non-viral vehicles. ${ }^{2-7}$ Despite their relatively low transfection activity, non-viral gene 25 delivery vehicles are expected to be preferred in terms of safety and clinical acceptance, with the cationic lipid systems ${ }^{8}$ and polymer-based platforms ${ }^{4-6,9,10}$ being the prime examples. Table S1† summarizes the main advantages and disadvantages of currently available transfection reagents/methods. ${ }^{1,10}$ An 30 ideal non-viral gene delivery system should be able to efficiently deliver the required DNA molecules into specific cell types with minimal cytotoxicity to ensure maximal expression of the gene of interest. ${ }^{11}$ However, to the best of our knowledge, current commercially-available transfection reagents fall considerably short of these criteria, especially when they are used for transfect hard-to-transfect (HTT) cells. ${ }^{12,13}$

HTT cells typically include primary cells, embryonic stem cells, and some immortalized cell lines, ${ }^{14}$ such as 3T3-L1 (murine preadipocyte), ${ }^{15}$ U937 (human monocyte), ${ }^{16,17}$ Jurkat (human T lymphocyte), ${ }^{6,7,9,18}$ HUVEC (human umbilical vein endothelial cells) and many others. ${ }^{13,19-22}$ Table S2† shows the transfection efficiency of HTT cells with plasmid (as an example) using commercially-available reagents. One of the reasons why HTT cells demonstrate poor uptake of foreign substances and plasmid relates to their low metabolic activity. ${ }^{21}$ Furthermore, these cells are usually highly sensitive to cellular perturbations evoked by the transfection reagents used and potentially leading to cytotoxicity and, eventually, cell death. ${ }^{8,23}$

Recently, cell-derived delivery systems have gained considerable attention, in view of their potential to deliver active cargo molecules to target sites with some extent of specificity. ${ }^{24}$ Indeed, a number of studies have explored the use of monocyte-derived drug delivery systems (DDS) in targeted delivery of 55 chemotherapeutics ${ }^{25,26}$ and plasmid DNA (pDNA) ${ }^{27}$ to tumors, or erythrocyte-derived DDS for prolonged blood circulation and targeted delivery of pDNA to the blood cells. ${ }^{28}$ It is postu- 
lated that the preservation of the cellular membrane components from the parent cells would confer to these cell-based DDS the ability to recognize specific target cells, possibly promoted by the ability to evade recognition by the body's immune system. ${ }^{29}$

Despite significant therapeutic interest, to date, the full potential of cell-based DDS in gene delivery remains largely unexplored. The key challenges to address include tedious and low-throughput isolation/production processes of cell-derived DDS (e.g. in the form of exosomes) and suboptimal gene loading/complexation techniques. ${ }^{30,31}$ Thus, while these systems seem advantageous in terms of intrinsic targeting properties, they have strong limitations regarding production yields and pDNA loading efficiencies. Hence, in order to overcome these limitations, we propose here the development of a novel hybrid gene delivery system that consists of both cellular membrane components for enhanced internalization by target cells and synthetic lipid components for enhanced pDNA loading.

In this study, we describe for the first time the development of micro-Cell Vesicle Technologies (mCVTs), a novel hybrid delivery platform obtained through the fusion of cationic lipids with cellular membranes (Fig. 1 and 2). To produce mCVTs, cell ghosts (CG) are first obtained by treating cells with a hypotonic aqueous solution, as described by Goh et $a .^{31}$ The hypotonic solution creates transient openings in the plasma membranes to allow the removal of the intracellular contents of the cells, while maintaining the integrity of the plasma membranes. CG derived from different cells suspended in aqueous medium are used to hydrate a dry thin film of DOTAP lipids and subsequently extruded through polycarbonate filters to provide mCVTs with a similar mean hydrodynamic size and zeta potential as compared to extruded DOTAP liposomes. In this study, we demonstrate that mCVTs are able to complex with plasmids and be internalized by target cells with minimal cytotoxicity. Interestingly, when mCVTs produced from different cells were compared in vitro, they showed significantly higher transfection efficiency for cell types from which the mCVTs were derived from.
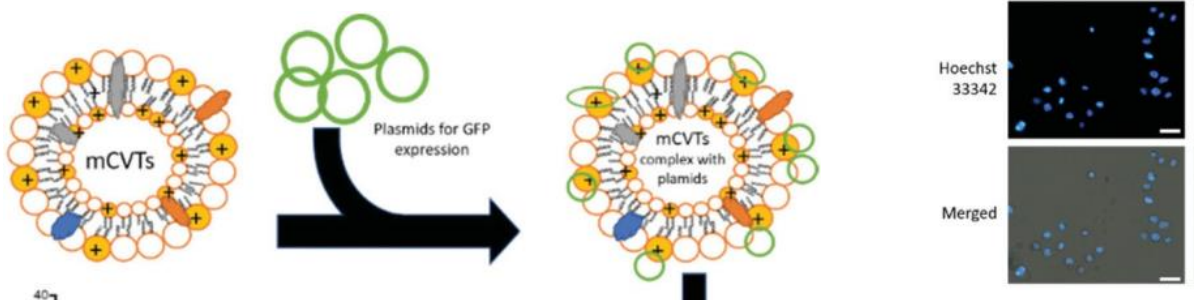
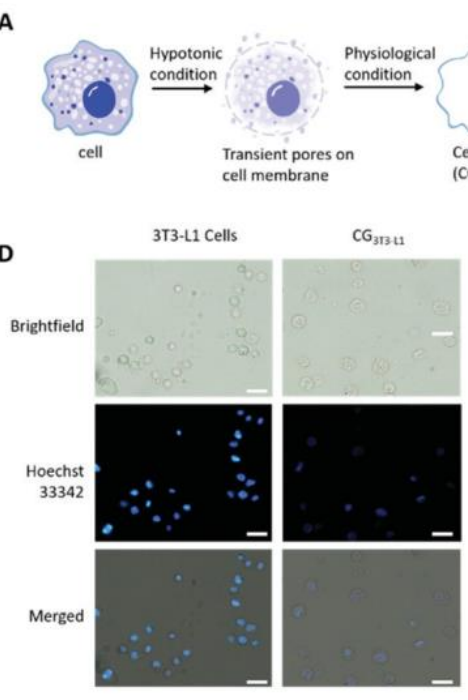

Fig. 3 Production and

CG). A Production scheme pressure. B. DNA gel electrophoresis image of 3T3-L1 cells (second lane) and CG (third lane). DNA ladder (first lane) represents $100 \mathrm{bp}$ DNA. C. Isolated DNA contents in 3T3-L1 cells and CG, respectively $(5 \times$ $10^{6}$ cells per CG). Data represent means $\pm \operatorname{SD}(n=3)$, ${ }^{* * *} P<$ 0.0001 . D. Fluorescence microscope images of 3T3-L1 cells and CG. Hoechst 33342 was used to stain nuclear contents. Scale bar indicates $100 \mu \mathrm{m}$. E. Cell proliferation tests of 3T3-L1 cells and CG. Data represent means $\pm \operatorname{SD}(n=3)$.

Fig. 1 Schematic overview of transfection of HTT cells using mCVTs. 
osmotic pressure to facilitate the formation of transient pores in the cell plasma membrane; at these conditions, the cytoplasm and organelles (e.g. nuclei, ribosomes, mitochondria) were postulated to leak out from the cells through these transient openings, which were subsequently resealed by replacing the hypotonic solution with an isotonic solution. ${ }^{31}$ At the same time, $0.1 \% \mathrm{w} / \mathrm{v}$ sucrose in PBS solution resulted in a sufficiently viscous solution to impede cell rupture or membrane flipping. To maintain the integrity of membrane proteins in $n$ CG, protease inhibitor cocktails were added to $\mathrm{CG}$ during the hypotonic solution treatment. As shown in Fig. $3 \mathrm{~B}$ and $\mathrm{C}$, more than $70 \%$ of the cellular DNA content was removed from 3T3-L1 cells in the process of CG production. This was further confirmed by staining the CG with Hoechst 33342, a fluorescent dye binding to nuclear DNA. The reduced fluorescence reinforces the notion that the DNA was largely removed through the $\mathrm{CG}$ production protocol (Fig. 3D). Noteworthy, the CG remained as spherical vesicles similar to the original cells, which may be indicative that the protocol did not destroy the overall structure of the plasma membranes. The size of CG appeared to be slightly larger than that of the cells, probably due to the interaction between the solutes and phospholipids in the plasma membrane after the physical permeabilization using the hypotonic solution. ${ }^{32}$

Removal of cellular and nuclear contents in the $\mathrm{CG}$ is an essential step to ensure production reproducibility and safety of the produced mCVTs with regards to minimalizing the amount of genetic material from parent cells that is transferred to target cells. To confirm that the cellular component of mCVTs is non-living, the non-proliferative nature of $\mathrm{CG}$ from 3T3-L1 ( $\left.\mathrm{CG}_{3 \mathrm{~T} 3-\mathrm{L} 1}\right)$ was investigated and compared to live cells. The number of $\mathrm{CG}_{3 \mathrm{~T} 3-\mathrm{L} 1}$ and 3T3-L1 cells in complete culture medium was plotted against time, as shown in Fig. 3E for 5 consecutive days. As anticipated, the 3T3-L1 cells showed a typical trend of cell proliferation, indicating normal growth of 3T3-L1 in cell culture. The number of cells started to decline at day 5 probably due to the fact that the culture became overcrowded. In comparison, the number of $\mathrm{CG}_{3 \mathrm{~T} 3 \mathrm{~L} 1}$ remained relatively constant throughout the 5 days, suggesting that $\mathrm{CG}$ produced through this protocol cannot proliferate and are no longer viable.

Production and characterization of mCVTs

Similar to liposome production, the lipid thin film method ${ }^{33}$ was used to produce mCVTs. DOTAP thin film was hydrated with CG to form large multilamellar liposomes encapsulating CG. This suspension was extruded and plasma membranes from the CG contained DOTAP lipids (as shown in Fig. 4A). Following a method previously established by our group, it was proven that, upon extrusion, the so-produced mCVTs consisted of the fusion of both lipids and CG membranes (see Fig. 5). The histogram in Fig. S1† demonstrates the size distribution profile of mCVTs derived from $\mathrm{CG}_{3 \mathrm{~T} 3-\mathrm{L} 1}\left(\mathrm{mCVT}_{3 \mathrm{~T} 3-\mathrm{L} 1}\right)$ and liposomes. Generally, the size distribution of mCVT was similar to liposomes. In order to demonstrate the robustness and versatility of this method across various cell lines, mCVTs were pro-

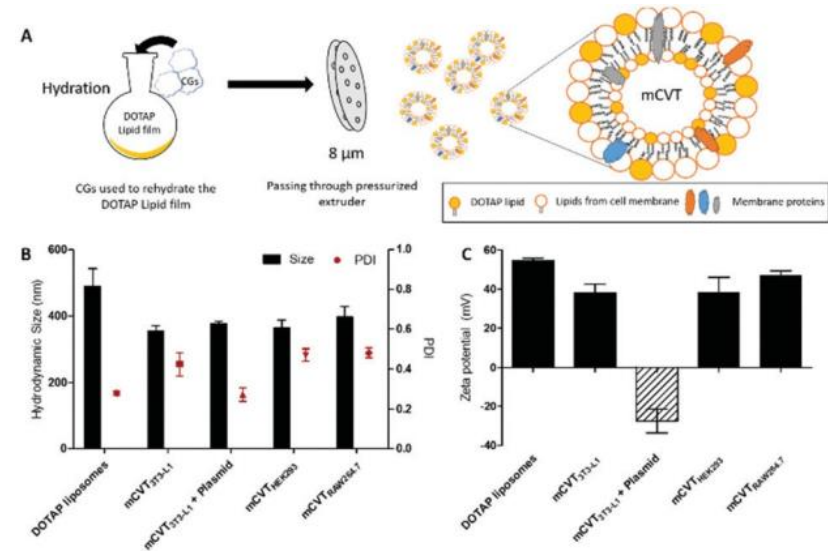

1

Fig. 4 Production and characterization of mCVTs. A. Schematic depiction of mCVTs, involving fusion of cell ghosts and liposomes made from thin film hydration. B. Hydrodynamic size of different mCVTs and $\mathrm{mCVT}_{3 T 3-\mathrm{L} 1}$ with plasmid compared to DOTAP liposome. The size of all mCVTs is smaller than $500 \mathrm{~nm}$. C. Zeta potential of different mCVTs and $\mathrm{mCVT}_{3 т 3-\mathrm{L} 1}$ with plasmid compared to DOTAP liposomes. The zeta potential of all mCVTs is above $+30 \mathrm{mV}$, except for $\mathrm{mCVT}_{3 \mathrm{ST}_{3} \mathrm{~L} 1}$ with plasmid DNA $(5 \mu \mathrm{g})$, showing negative zeta potential due to the interaction with plasmid DNA. Data represent means $\pm \operatorname{SD}(n=3)$.
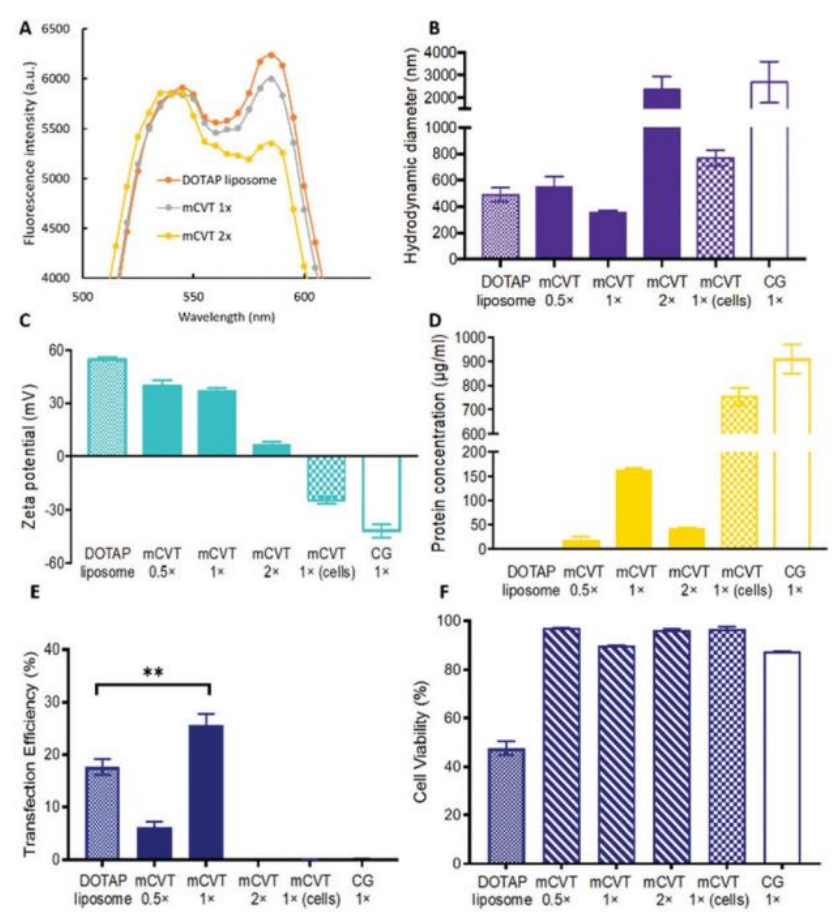

Fig. 5 Proof-of-fusion of $\mathrm{mCVT}_{3 т 3-L 1}$. Fluorophores are in close proximity in liposomes, while fusion of DOTAP liposomes with CG increases the distance between two fluorophores. A. FRET analysis of NBD and rhodamine fluorophores of DOTAP liposome and mCVTs. Upon exciting at $460 \mathrm{~nm}$, the energy of emission spectrum for NBD excited rhodamine, resulting in the emission of rhodamine at $585 \mathrm{~nm}$. The emission of rhodamine was higher compared to mCVT $1 \times$ and mCVT 2x, suggesting fusion between DOTAP liposomes and $\mathrm{CG}_{3 T 3-\mathrm{L} 1}$. Hydrodynamic size (B) zeta potential $(C)$ and protein concentrations (D) of DOTAP liposome, mCVT $0.5 \times$, mCVT $1 \times$, mCVT $2 \times$, DOTAP + cells and CG. Transfection efficiency (E) and cell viability (F) of 3T3-L1 cells upon transfection. $n=3,{ }^{* *} p<0.01$. 
1 duced using CG derived from different cell lines, such as some HTT cells (i.e. 3T3-L1) and non-cancerous cells (i.e. HEK293 and RAW264.7). As shown in Fig. 4B, the hydrodynamic size and PDI of various mCVTs was typically in the range of $400 \mathrm{~nm}$ (with PDI 0.4), similar to DOTAP liposomes (PDI 0.2) produced using the same extrusion method. In Fig. 4C, similar zeta potential values (around $+30 \mathrm{mV}$ to $+40 \mathrm{mV}$ ) were observed for different formulations, indicating that this method is generally applicable to CG generated from different cell lines. Interestingly, when mCVT $_{3 \mathrm{~T} 3-\mathrm{L} 1}$ was complexedwith pDNA, the size distribution remained similar to that of the non-complexed mCVTs, while the overall zeta potential became more negative (Fig. S2†), which may be indicative for a successful complexation (as the negatively charged pDNA neu- tralized the positively charged mCVTs).

To demonstrate the fusion between the lipids and CG, a fluorescence resonance energy transfer (FRET) assay was conducted as reported previously. ${ }^{31}$ Two formulations, made from $2.5 \mathrm{mg}$ of lipids and either $1 \times 10^{7} 3 \mathrm{~T} 3-\mathrm{L} 1 \mathrm{CG}$ or $2 \times 10^{7} 3 \mathrm{~T} 3-$ L1 CG, were used to produce mCVTs and their rhodamine emis-

sion intensities were compared against those observed in the DOTAP liposomes. As depicted in Fig. 5A, in the absence of CG fusion, both the NBD and rhodamine fluorophores in the liposomal formulation were in close proximity, thus DOTAP liposomes alone had the highest observed rhodamine emission intensity at $585 \mathrm{~nm}$. When CG were fused with DOTAP liposomes, cellular membranes from CG were randomly inserted between the two fluorophores, pushing apart the NBD and rhodamine fluorophores, thus resulting in a reduction of the observed emission intensity at $585 \mathrm{~nm}$. Consistent with this observation, the emission intensity of rhodamine decreased further when twice the amount of CG was used for mCVTs production (Fig. 5A). Altogether, the results confirmed the fusion between cationic lipids and $\mathrm{CG}$, rather than the encapsulation of CG into liposomes or vice versa.

mCVTs produced from various amount of CG (Table S3†) were characterized by measuring the hydrodynamic size, zeta potential and protein concentration. Increasing the CG quantity in the mCVTs formulation reduced the hydrodynamic size of the prepared mCVTs (from half amount, i.e. mCVT $0.5 \times$, to full amount, i.e. mCVT $1 \times)$, till a hydrodynamic mean size of about $400 \mathrm{~nm}$ was observed (in mCVT $1 \times$ ). When more CG were added (double amount, i.e. mCVT $2 \times$ ), the hydrodynamic size of the mCVTs increased, possibly due to excess of CG leading to a higher propensity to aggregate (Fig. 5B). Moreover, the size of mCVTs was unaffected after the complexation, suggesting that the plasmid DNA was complexed to mCVTs by electrostatic interactions and did not result in aggregation of mCVTs. The zeta potential was generally positive due to the incorporation of cationic DOTAP. Since CG have a negative zeta potential as inherited from the parent cells, the overall positive charge of mCVTs confirms the successful incorporation of DOTAP into the cell membrane of CG. The positive zeta poten- tial decreased when a higher concentration of $\mathrm{CG}_{3 \mathrm{~T} 3-\mathrm{L} 1}$ was

used (e.g. with mCVT $2 \times$ ), providing additional evidence that DOTAP lipids were indeed inserted into the plasma mem- branes. The greatest decrease in zeta potential was observed when $1 \times 10^{7} 3 \mathrm{~T} 3-\mathrm{L} 1$ cells were used to interact with DOTAP lipids (mCVT $1 \times($ cells)), showing a negative zeta potential. These decreases in zeta potential are likely due to the increasing amounts of proteinaceous materials or other cellular components (e.g. nuclear content) from the cells relative to the amounts of cationic lipids (Fig. 5C). Once again, this highlights the importance of producing $\mathrm{CG}$ prior to mCVTs production as the interaction of cationic lipids with cells can

immediately result in formation of aggregates instead of mCVTs (Fig. 5D). CG were also not a suitable construct for transfection as no transfection was observed with cells treated with the CG alone. This is likely due to the inability of CG (which have a negative zeta potential) to complex with plasmid (Fig. 5E). Interestingly, mCVT $2 \times$ did not show a higher protein concentration or better transfection performance compared to mCVT $1 \times$, despite the fact that only half of the amount of $\mathrm{CG}$ was used for the production of the latter preparation (Fig. 5D and E). One plausible explanation is a lower protein yield in case of mCVT $2 \times$ due to the formation of protein aggregates during the extrusion process, as the higher amount of CG leads to much more clogging of the extrusion membranes, which translated into substantially more protein loss.

\section{Cell ghosts (CG) are required for production of mCVTs}

To investigate the effect of cellular contents on the production yield and the transfection efficiency of a model gene (enhanced green fluorescence protein, eGFP), $1 \times 10^{7}$ of 3T3-L1 cells were used for mCVTs production and compared with mCVTs produced from CG. mCVT $1 \times$ (cells) showed submicron size with a negative net zeta potential and high protein concentration, which did not contribute to the in vitro gene delivery to cells. Similar to $\mathrm{mCVT} 2 \times$ from CG, protein aggregation happened during the production process with mCVT $1 \times$ (cells), and the bulk of proteins from both plasma membranes and intracellular contents hindered the interaction with cationic DOTAP, resulting in a net negative charge with no transfection. Conversely, the $\mathrm{mCVT} 1 \times$ (from CG rather than whole 40 cells) showed to be a suitable combination of cellular membrane (and proteins) and lipid charges to form mCVTs, which translated into a high transfection efficiency and viability for target HTT cells (Fig. 5E and F) in comparison to DOTAP liposomes or CG alone. Altogether, these results emphasize the 45 importance of the cellular component from the CG in providing plasmid transfection in HTT cells, as well as in masking the cytotoxicity of cationic liposomes.

Presence of plasma membrane components improves the cellular uptake of mCVTs by HTT cells

In order to understand how plasma membrane components in mCVTs affect uptake and transfection of target HTT cells, cyanine 5.5 (Cy5.5) was used to label the proteins in the outer 55 cellular membrane of $\mathrm{CG}_{3 \mathrm{~T} 3-\mathrm{L} 1 \text {, which were used to produce }}$ $\mathrm{mCVT}_{3 \mathrm{~T} 3-\mathrm{L} 1}$; and NBD labelled DOTAP lipids were used for the formation of the cationic liposomes. 
Fig. 6A shows the fluorescence profiles by flow cytometry of $\mathrm{CG}_{3 \mathrm{~T} 3-\mathrm{L} 1}$, DOTAP liposomes and $\mathrm{mCVT}_{3 \mathrm{~T} 3-\mathrm{L} 1}$ after labelling. The presence of both NBD and Cy5.5 in mCVT $_{3 \mathrm{~T} 3-\mathrm{L} 1}$ indicates the incorporation of both DOTAP lipids and 3T3-L1 cell membrane components in $\mathrm{mCVT}_{3 \mathrm{~T} 3-\mathrm{L} 1}$. This is in agreement with our FRET results (Fig. 5A), confirming that $\mathrm{mCVT}_{3 \mathrm{~T} 3-\mathrm{L} 1}$ is a hybrid system consisting of these two components.

Subsequently, the fluorescence-labelled DOTAP liposomes, $\mathrm{CG}_{3 \mathrm{~T} 3-\mathrm{L} 1}$ and $\mathrm{mCVT}_{3 \mathrm{~T} 3-\mathrm{L} 1}$ were added to 3T3-L1 cells to study their uptake profile (Fig. 6B). DOTAP liposomes were taken up by the cells at a slower rate as compared to $\mathrm{CG}_{3 \mathrm{~T} 3-\mathrm{L} 1}$, as after 1 hour of incubation with liposomes, only a small percentage of cells were positive for NBD $(<10 \%)$, whereas $>90 \%$ of cells were positive for $\mathrm{Cy} 5.5$ after 1 hour of incubation with $\mathrm{CG}_{3 \mathrm{~T} 3-\mathrm{L} 1}$. For the cellular uptake of $\mathrm{mCVT}_{3 \mathrm{~T} 3-\mathrm{L} 1}$, it appeared that our mCVTs (hybrid system) were being taken up by cells at a rate comparable to $\mathrm{CG}_{3 \mathrm{~T} 3-\mathrm{L} 1}$ ( plasma membrane particles), and faster than DOTAP liposomes (fully synthetic lipid vesicles). Thus, as mCVTs represent a hybrid system of cationic lipids and cell membrane components, we postulate that they facilitate the internalization of plasmid and transfection through the following mechanisms: (1) complexation of plasmid DNA with cationic lipids allows mCVTs to carry the plasmid DNA into 3T3-L1 cells, as naked plasmid is unable to enter cells on its own; (2) the positive charges of cationic lipids facilitate the interaction of mCVTs with the negatively charged cell membranes of 3T3-L1 cells; (3) the surface properties (from plasma membrane components) on mCVTs further promote the interaction with target cells; and (4) efficient cellular uptake of membrane components of mCVTs (as indicated by the efficient uptake of CG) facilitates the delivery of plasmid

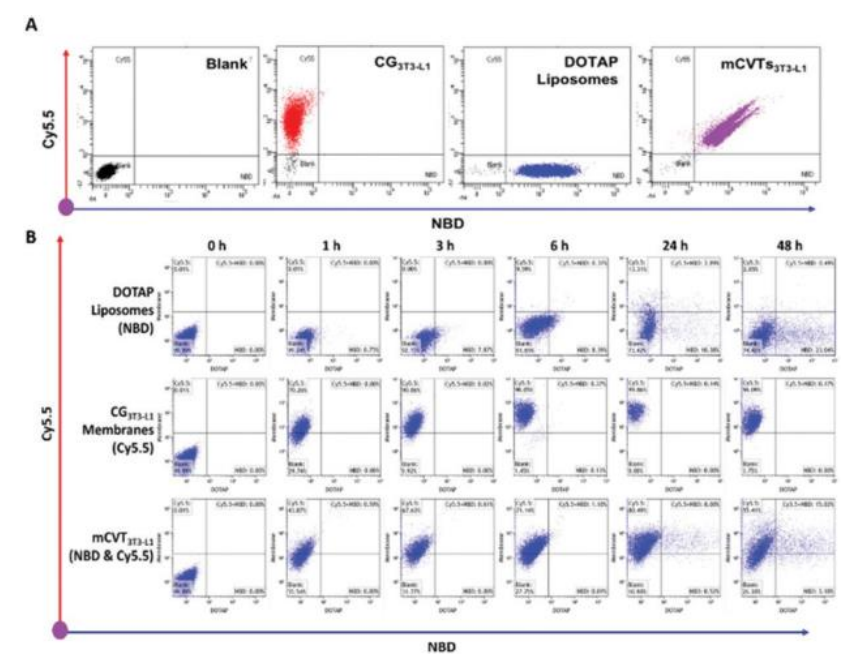

Fig. 6 Uptake of $\mathrm{mCVT}_{3 \mathrm{~T} 3-\mathrm{L} 1}$, DOTAP liposome and $\mathrm{CG}_{3 \mathrm{~T} 3 \mathrm{-L} 1}$ by 3T3-L1 cells. Fluorescently labelled components (NBD-DOTAP and Cy5.5$\mathrm{CG}_{3 T 3-\mathrm{L} 1}$ ) were used for production of mCVTs. A. Dot plots of $\mathrm{CG}_{3 T 3-L 1}$, DOTAP liposome and $\mathrm{mCVT}_{3 \mathrm{~T} 3 \mathrm{~L} 1}$ for flow cytometry analysis. Black: blank; red: Cy5.5; blue: NBD; purple: both Cy5.5 and NBD. B. Uptake of DOTAP liposome, $\mathrm{CG}_{3 T 3-\mathrm{L} 1}$ and $\mathrm{mCVT}_{3 T 3-\mathrm{L} 1}$ into 3T3-L1 cells. Analysis was done for 1, 3, 6, 24 and 48 h. Blank: green; red: Cy5.5; blue: NBD; purple: both Cy5.5 and NBD.
DNA into the cells. Taken together, the presence of membrane components in mCVTs facilitates transfection of plasmid DNA into HTT cells.

mCVTs display superior transfection capability and reduced cytotoxicity

Having characterized mCVT $_{3 \mathrm{~T} 3-\mathrm{L} 1}$ and demonstrated their cellular uptake, we next investigated plasmid delivery efficiency of mCVT $_{3 \text { T3-L1 }}$ using 3T3-L1 cells and eGFP as model plasmid. Transfection efficiency of $\mathrm{mCVT}_{3 \mathrm{~T} 3 \mathrm{~L} 1}$, was compared with a widely used commercial transfection reagent, Lipofectamine 3000 (LF3000). When $5 \mu \mathrm{g}$ of the total plasmids were used, the maximal transfection efficiency (expressed as number of cells transfected) of 3T3-L1 cells, as a HTT cell line, using LF3000 was about $15 \%$ at $10 \mu \mathrm{l}$ (Fig. 7A). Higher amounts of LF3000 resulted in a lower transfection efficiency attributable to a higher cytotoxicity (Fig. 7B). In comparison, the maximal transfection efficiency of 3T3-L1 cells by mCVT $_{3 \mathrm{~T} 3-\mathrm{L} 1}$ (equivalent to $1.25 \mathrm{mg} \mathrm{ml}^{-1}$ of DOTAP lipids, and about $80 \mu \mathrm{g} \mathrm{ml}^{-1}$ of $\mathrm{CG}$ proteins) reached $30 \%$ at $80 \mu \mathrm{l}$ ). Because $\mathrm{mCVT}_{3 \mathrm{~T} 3-\mathrm{L} 1}$ started to induce detectable cytotoxicity in 3T3-L1 cells at the

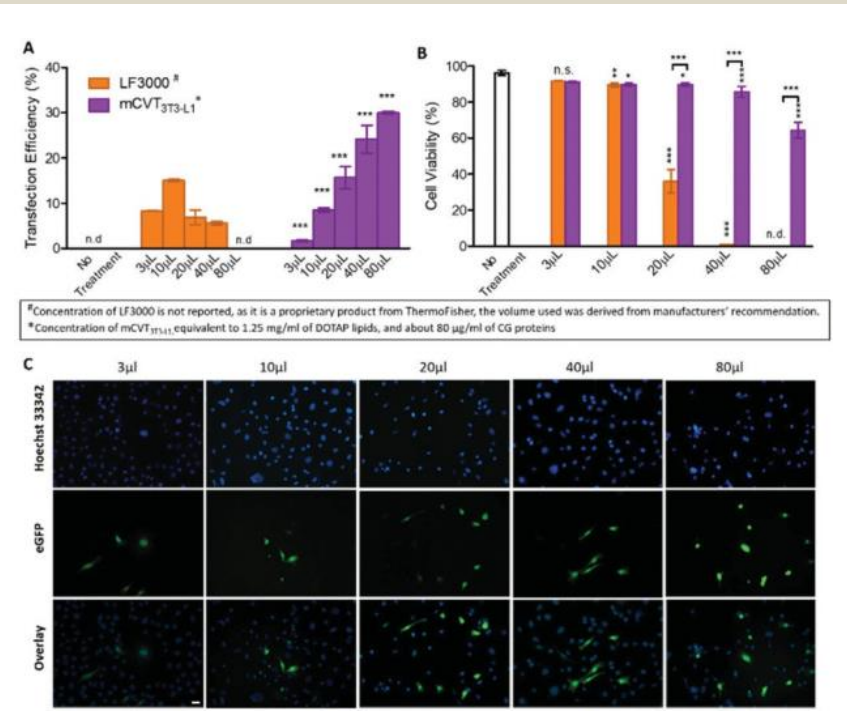

Fig. 7 Transfection efficiency and cytotoxicity of mCVTs. A. Transfection efficiency of LF3000 and mCVT 3 3T3-L1 into 3T3-

L1 cells. 5 different amounts $(3 \mu \mathrm{l}, 10 \mu \mathrm{l}, 20 \mu \mathrm{l}, 40 \mu \mathrm{l}$ and $80 \mu \mathrm{l})$ of LF3000 were used to transfect $5 \times 10^{5}$ number of 3T3-L1 cells, with the same corresponding doses of mCVTs chosen for the same transfection experiments for comparison. Cells without treatment were used as the control. ${ }^{* * *} P<0.001$ (for comparison to respective amount of LF3000) B. Cell viability of 3T3-L1 after transfection using LF3000 and 50 $\mathrm{mCVT}_{3 \mathrm{~T} 3 \mathrm{~L} \text {.1 }}$. Cells without treatment were used as the control. n.d. and n.s. indicated no detectable transfection and not significantrespectively. Data represented means $\pm \operatorname{SD}(n=3),{ }^{*} P<0.05^{* *} P<0.01,{ }^{* * *} P<$ 0.001. C. Images of transfection cells using different doses of $\mathrm{mCVT}_{3 т 3-}$ L1. The transfected cells expressed eGFP (green). Hoechst

33342 (blue) was used to visualize the nucleus. Scale bar represents $100 \mu \mathrm{m}$. ${ }^{a}$ Concentration of LF3000 is not reported, as it is a proprietary product from ThermoFisher, the volume used was derived from manu-

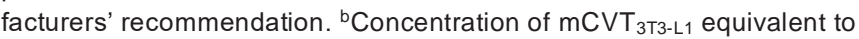
$1.25 \mathrm{mg} \mathrm{ml}^{-1}$ of DOTAP lipids, and about $80 \mu \mathrm{g} \mathrm{ml}^{-1}$ of CG proteins. 
amount of $80 \mu 1$, we considered $40 \mu \mathrm{l}$ of $\mathrm{mCVT}_{3 \mathrm{~T} 3-\mathrm{L} 1}$ as the optimal transfection dose that reached significantly higher transfection efficiency $\left(25 \pm 3.1 \%\right.$ for mCVT $_{3 \mathrm{~T} 3-\mathrm{L} 1}$ vs. $15 \pm 0.3 \%$ for LF3000, $p<0.001$ ) with marginal cytotoxicity.

The mCVT $_{3 \mathrm{~T} 3-\mathrm{L} 1}$ transfected cells were also monitored using fluorescence microscopy imaging. The cell nuclei were visualized using Hoechst 33342 (Fig. 7C). In agreement with Fig. 7A, an increase in intracellular eGFP signal was observed when higher doses of $\mathrm{mCVT}_{3 \mathrm{~T} 3-\mathrm{L} 1}$ were used. Overall, $\mathrm{mCVT}_{3 \mathrm{~T} 3 \mathrm{~L} 1}$

were about twice as effective as LF3000 with regards to transfection efficiency and yet exhibited only moderate cytotoxicity compared to LF3000, indicating their significant advantage for the transfection of HTT cells.

Next, we explored whether the use of mCVTs for transfection was associated with some extent of specificity towards the target cells. mCVTs were produced using CG from different cell types, including 3T3-L1 cells, HEK293 cells and RAW264.7 macrophages, and were tested for their ability to transfect our model gene into 3T3-L1 cells. LF3000, CG and DOTAP liposomes were used as controls (Fig. 8A). The dosage of LF3000 $(10 \mu 1)$, DOTAP liposomes $(40 \mu 1)$ and mCVTs $(40 \mu 1)$ were selected based on maximal transfection efficiency and minimal cytotoxicity results (see Fig. 8B).

Cells treated with $\mathrm{CG}_{3 \mathrm{~T} 3-\mathrm{L} 1}$ and plasmid showed no detectable transfection due to the inability of anionic $\mathrm{CG}$ to complex with plasmid DNA. Although it was shown that CG could be taken up by cells without complexation, CG were not able to facilitate the entry of plasmid DNA into 3T3-L1 cells. DOTAP liposomes exhibited a similar transfection efficiency as LF3000 at a dose of $40 \mu \mathrm{l}$ but they caused high cytotoxicity due to their highly cationic nature. Conversely, all mCVTs displayed minimal cytotoxicity towards 3T3-L1 cells and they showed either comparable (in the case of $\mathrm{mCVT}_{\mathrm{HEK} 293}$ and $\mathrm{mCVT}_{\mathrm{RAW} 264.7}$ ) or superior transfection efficiency (in the case of mCVT $\left._{3 \mathrm{~T} 3-\mathrm{L} 1}\right)$ when compared to the commercial LF3000 and DOTAP liposomes. This is likely due to the presence of plasma membrane components (from CG) that rendered mCVTs less

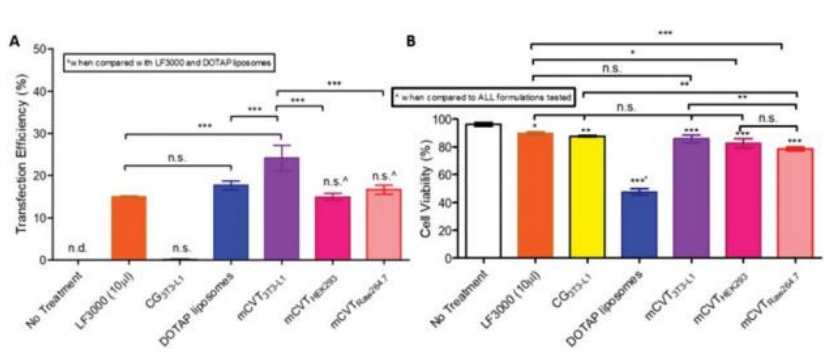

Fig. 8 Transfection efficiency and cytotoxicity of different mCVTs on 3T3-L1 cells after 24 hours incubation. A. Transfection efficiency of LF3000 (10 $\mu \mathrm{l}), \mathrm{CG}_{3 T 3-\mathrm{L} 1}$, DOTAP liposomes $(40 \mu \mathrm{l})$ and different mCVTs $(40 \mu \mathrm{l})$ on $3 \mathrm{~T} 3-\mathrm{L} 1$ cells. B. Cell viability of 3T3-L1 cells after transfection with LF3000, $\mathrm{CG}_{3 T 3-\mathrm{L} 1}$, DOTAP liposomes and different mCVTs. n.d. and n.s. indicated no detectable transfection, and not significant respectively. Data represented means $\pm \operatorname{SD}(n=3),{ }^{*} P<0.05,{ }^{* *} P<0.01$ and ${ }^{* * *} P<0.001$. cytotoxic. This again highlights the advantage of using mCVTs as transfection agent.

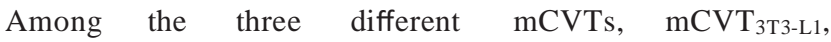
mCVT $_{\text {HEK293 }}$ and mCVT $_{\text {RAW264.7, }}$ mCVT $_{3 T_{3}-\mathrm{L} 1}$ showed the highest transfection efficiency (Fig. 8A). Since not all mCVTs (dose dependent transfection efficiency of different mCVTs and respective cell viability can be found in Fig. S3†) were able to transfect 3T3-L1 to a similar extent, we reasoned that the presence of plasma membrane components of 3T3-L1 cells may have induced specificity towards 3T3-L1 cells. This homotypic targeting behaviour suggests that, when formulating mCVTs, the selection of the appropriate cell line is crucial in improving the overall transfection efficiency.

In this study, 3T3-L1 cells, a murine preadipocyte cell line, were used as a quintessential example of HTT cells. mCVTs produced from 3T3-L1 CG were able to improve the transfection efficiency to approximately $30 \%$ while maintaining nearly $90 \%$ of cell viability upon transfection. This is in contrast with other commercial transfection reagents (for example LF3000), which have limited transfection efficiency $(\approx 15 \%)$, and high cytotoxicity when used at similar concentrations. Furthermore, mCVTs were found to maintain a relatively high transfection efficiency $(\approx 20 \%)$, even under serum condition (Fig. S4†). This highlights the additional advantage of using mCVTs as potential ex vivo or in vitro transfection reagent, as serum can be added during the process to nourish and protect the sensitive cell lines (i.e. primary cells).

By studying mCVTs and the extent of cellular uptake when different parent cells were used, we identified several key insights. First, the transfection efficiency of non-viral-based gene delivery systems is typically influenced by the zeta potential of the overall complexes, comprising of vector and genetic load. Data from the literature recommend that a higher positive zeta potential is preferred to increase gene transfection, ${ }^{34,35}$ but that cell viability is strongly limiting the degree of transfection. Surprisingly, we did not observe such a correlation for mCVTs. In our study, mCVTs had an overall negative zeta potential between -30 to $-40 \mathrm{mV}$ after complexing with the plasmid, and yet they were able to successfully transfect the HTT cell lines. We speculate that the plasma membrane components present in mCVTs likely contribute to improving the transfection efficiency in case of HTT cells. Second, the use of different precursor cells in the production of mCVTs affects the overall transfection efficiency and cytotoxicity profiles. Interestingly, we observed a certain degree of "like-to-like" selectivity of mCVTs 3T3-L1 $_{3}$ towards 3T3-L1 cells, as mCVTs 3 T3-L1 transfect 3T3-L1 cells more efficiently than the mCVTs produced from other cell lines (similar results were 50 obtained when HEK cells were transfected with mCVT $_{\text {HEK293 }}$ (Fig. S5†)). We postulate that mCVTs are preferentially taken up by the cells of the same origin to some degree, likely due to the conservation of surface recognition moieties during the production of mCVTs. These fingerprints are later used as recognition moieties for homotypic targeting. A similar phenomenon was reported for other cell-based DDS (i.e. exosomes). Sancho-Albero et al. ${ }^{36}$ demonstrated a selective uptake 
of exosomes derived from mesenchymal stem cells (MSC) by other MSCs in a co-culture system. Likewise, the results reported by Hazan-Halevy et al. ${ }^{37}$ indicated that exosomes derived from B lymphocytes were efficiently internalized by other B lymphocytes and not by other immune cells. All these studies suggest a role for surface recognition moieties in specifically recognising the cell types used for the production of the mCVTs.

However, it is noteworthy that the nature of the selective targeting of cell-based nanovesicles (e.g. exosomes) is still largely debatable: some studies (like those reported above) have demonstrated homotypic targeting, while others highlighted that cell-based vesicles could be used to target different target cells, such as nanovesicles derived from U937 monocytes showing targeting properties towards inflammatory or cancer tissue. ${ }^{25,26}$

Therefore, the selective nature of mCVTs may not be solely towards precursor cells. Instead, we found that it was possible to use $\mathrm{mCVT}_{3 \mathrm{~T} 3-\mathrm{L} 1}$ to transfect other HTT cells such as keratocytes (HaCaT) (Fig. S6†). One plausible explanation is that mCVTs possess receptor/ligand molecules, acquired from precursor cells, which are responsible for cell-cell interactions. In this regard, 3T3-L1 cells are known to express a basal level of inflammatory markers such as MCP-1 and ICAM-1, and other integrin molecules which are responsible for cell adhesions. ${ }^{38}$ When passing down to $\mathrm{mCVT}_{3 \mathrm{~T} 3-\mathrm{L} 1}$, these cell surface markers may hence enable $\mathrm{mCVT}_{3 \mathrm{~T} 3-\mathrm{L} 1}$ to interact with other cells with corresponding receptors/ligands, leading to better uptake of mCVTs and, in this case, improved transfection. Our results thus lay the groundwork to further explore the possible interactions between membrane proteins across different cell types. Overall, we have demonstrated here the advantages of the presence of plasma membrane components in mCVTs in order to enhance selectivity and cellular uptake, as well as to increase the overall transfection efficiency of HTT cells. Furthermore, we demonstrated the "tunability" of producing mCVTs from various precursor cells according to the desired transfection outcome.

As a hybrid system of plasma membrane components and lipids, various lipids can be added to or replace the cationic lipids, in order to confer new functional- ities to mCVTs. This flexibility could be further exploited for the addition of fluorophores conjugated to lipids for visualization or tracking purposes, or for incorporation of magnetic nanoparticles for cell manipulation or even for attaching additional cell targeting ligands to achieve improved target cell selectivity.

\section{Conclusions}

In this study, we have established a protocol for $\mathrm{CG}$ production by varying the osmotic pressure, where CG were shown to be non-proliferative, with most nuclear material removed. Using cationic lipids and CG, we constructed a hybrid plasmid delivery system comprising both plasma membrane and cationic lipid components, which we termed mCVTs. We have also shown that mCVTs have similar characteristics and ability to 1 complex with plasmid DNA as cationic liposomal systems. Furthermore, mCVTs are superior to cationic liposomes and the commercial transfection reagent LF3000, in terms of transfection efficiency and cytotoxicity. Due to the chimeric nature 5 of mCVTs, comprising both plasma membrane components and cationic lipids, mCVTs were more efficiently taken up by target cells. Furthermore, mCVTs, being cell-derived formulations, confer flexibility in changing the cell-based components depending on the target cells or tissues and do not 10 require heavy capital investment into sophisticated pieces of equipment. Hence, the whole process of production of mCVTs is ideal for quick in-house testing and also amenable for upscaling. Taken together, the successful development of mCVTs provides a basis for the in vitro delivery of plasmids and other nucleic acids, especially for HTT cells, paving the way for gene delivery into a broader range of cell types.

\section{Materials \& methods}

Materials

1,2-Dioleoyl-3-trimethylammonium-propane (DOTAP), 1-oleoyl2-[6-[(7-nitro-2-1,3-benzo-xadiazol-4-yl)amino]hexanoyl]-3-trimethylammonium propane (NBD-DOTAP) and 1,2-dioleoyl-snglycero-3-phosphoethanolamine- $N$-(lissamine rhodamine B sulfonyl) (Liss Rhodamine) were purchased from Avanti Polar Lipids (Alabama, United States) and kept at $-20{ }^{\circ} \mathrm{C}$. Chloroform was used to dissolve the lipids. Lipofectamine 3000 (LF3000), Hoechst 33342 nuclear stain, trypan blue stain solution $\left(\mathrm{Hyclone}^{\mathrm{TM}}\right.$ ) and bicinchoninic acid (BCA) protein assay and eGPF expressing plasmid was purchased from ThermoFisher Scientific (Massachusetts, United States). $8 \mu \mathrm{m}$ membrane filters were purchased from Merck Millipore 35

(Massachusetts, United States) and used as supplied. Cyanine $5.5 \mathrm{~N}$-hydroxysuccinimide (NHS) monoester dyes were purchased from Kerafast (Boston, United States) and used as per manufacturer's recommendations. Polybead ${ }^{\circledR}$ Microspheres $10 \mu \mathrm{m}$ were obtained from Polysciences, Inc. (Pennsylvania, United States).

\section{Cell culture}

3T3-L1 cells, a fibroblast-like mouse preadipocyte cell, were cultured in Dulbecco's high glucose modified Eagle's medium/ high glucose (DMEM/high glucose, HyClone ${ }^{\mathrm{TM}}$ ) containing $10 \% \mathrm{v} / \mathrm{v}$ foetal bovine serum (FBS, HyClone ${ }^{\mathrm{TM}}$ ) and $1 \% \mathrm{v} / \mathrm{v}$ MEM non-essential amino acid solution (NEAA, Life Technologies, California, United States). HEK293 cells (human embryonic kidney cells) and RAW264.7 cells (mouse macrophages), were cultured in DMEM/high glucose supplemented with $10 \%$ FBS. Cells were cultured in a humidified incubator maintained at $5 \% \mathrm{CO}_{2}$ and $37{ }^{\circ} \mathrm{C}$.

Production of cell ghosts (CG)

CG were produced via changing osmotic pressure using neutral phosphate buffer saline (PBS, $\mathrm{pH}$ 7.4) with $0.1 \% \mathrm{w} / \mathrm{v}$ 
sucrose (PBS/sucrose). Cells were harvested and incubated in $0.25 \times$ PBS (diluted by sterile MilliQ water) at room temperature for 24 hours, followed by incubating CG in $0.1 \times \mathrm{PBS} /$ sucrose. CG were harvested in $1 \times \mathrm{PBS} /$ sucrose and stored at $4{ }^{\circ} \mathrm{C}$ for further experiments. Protease inhibitor cocktail (Abcam, Cambridge, United Kingdom) was added throughout the experiment. The presence of nuclei material was checked by staining CG with Hoechst 33342. And remaining DNA material in CG was isolated using Qiagen DNeasy blood and tissue kit (Hilden, Germany) for DNA gel electrophoresis according to manufacturer's instruction.

\section{Cell ghosts (CG) viability test}

The same number of 3T3-L1 cells and CG were prepared in DMEM high glucose culture medium with $10 \%$ FBS and $1 \%$ NEAA. The samples were collected, and total cell or CG count were determined by staining with trypan blue solution and counting via a cell counting chamber on days 1, 2, 3, 4 and 5 .

\section{Production of mCVTs}

$2.5 \mathrm{mg}$ of DOTAP was dissolved in $1 \mathrm{ml}$ of chloroform in a $5 \mathrm{ml}$ round bottom flask. A dry thin lipid film was formed on the round bottom flask under vacuum at $35{ }^{\circ} \mathrm{C}$ for at least 1 hour. CG suspended in PBS were added into the dry lipid film and were subsequently agitated by brief sonication. The mixture was passed through $8 \mu \mathrm{m}$ membrane filters using a liposome extruder (Genizer ${ }^{\mathrm{TM}}$, California, United States) for 3 times. Samples were taken for the measurement of hydrodynamic size, poly dispersity index (PDI) and zeta potential. Liposomes used in this study were produced, in the same manner, with same formulation without adding CG.

\section{mCVTs characterization}

All hydrodynamic size and zeta potential measurements were determined at $25{ }^{\circ} \mathrm{C}$ by dynamic light scattering (DLS) on a Zetasizer Nano ZS (Malvern, UK). Characterization data were analyzed using software supplied with the Zetasizer and the results from at least three different measurements per run for more than 10 runs carried per sample were presented. Protein concentration was determined using (bicinchoninic acid assay) BCA protein assay for each sample.

\section{Proof-of-fusion assay}

A fluorescence resonance energy transfer (FRET) assay was conducted. Liposome and $\mathrm{mCVT}_{3 \mathrm{~T} 3-\mathrm{L} 1}$ were prepared as described previously ${ }^{31}$ with the addition of 0.1 mol\% NBDDOTAP and lissamine rhodamine lipid. To prove the incorporation of cell membrane components from $\mathrm{CG}_{3 \mathrm{~T} 3-\mathrm{L} 1}$ to $\mathrm{mCVT}_{3 \mathrm{~T} 3-\mathrm{L} 1}$, various concentrations of CG $\left(0.5 \times 10^{7}, 1 \times 10^{7}\right.$ and $2 \times 10^{7}$ of $\mathrm{CG}_{3 \mathrm{~T} 3-\mathrm{L} 1}$ ) were used to produce $\mathrm{mCVT}_{3 \mathrm{~T} 3 \mathrm{~L} 1}$ while the amount of DOTAP used remain unchanged. The liposome and mCVTs were then measured at $460 \mathrm{~nm}$ using a microplate reader and the emission spectra from $300 \mathrm{~nm}$ to $700 \mathrm{~nm}$ were recorded.
Transfection of 3T3-L1 using LF3000 and mCVTs

3T3-L1 cells with $60-70 \%$ confluency were ready for transfection. 3T3-L1 cells were transfected with plasmid DNA containing pEZX-MR04 (miRNA overexpression scrambled control clone, GeneCopoeia) using Lipofectamine 3000 according to the manufacturer's instruction. For transfection of 3T3-L1 using mCVTs, plasmid DNA (fixed complexation ratio of $5 \mu \mathrm{g}$ / $40 \mu 1)$ was incubated with various concentration of mCVTs at room temperature for 10 minutes, followed by incubating the 10 cells with serum-free medium. The final amount of pDNA used in corresponding experiments (LF3000 and mCVTs) were the same. After incubating in serum free medium for 6 hours, the cells were rinsed with PBS and cultured under complete medium with $1 \%$ penicillin-streptomycin (Hyclone, Utah, 15 United States). Propidium iodide (PI) was added to determine cell viability, the viable cells with intact cell membrane will be able to exclude the dye, whereas, dead/dying cells will be stained. The percentage transfection efficiency and cell viability were determined by detecting eGFP (reporter of the 20 plasmid) and PI (as cell viability dye), respectively, using BD LSR Fortessa flow cytometry analyser (New Jersey, United States). The untreated cells were used as control. All fluorescence images were taken using Olympus BX51 fluorescence microscope (Tokyo, Japan).

Flow cytometry analysis of mCVTs and liposomes

Fluorophore labelled DOTAP liposomes were made by incorporating $0.1 \mathrm{~mol} \%$ NBD-DOTAP in the lipid formulation of 30 DOTAP liposome. Cyanine 5.5 (Cy5.5) $N$-hydroxysuccinimide (NHS) monoester was used for the labelling of membrane protein of CG. The fluorophore labelled CG were then used to produce mCVTs. DOTAP liposomes and mCVTs were adsorbed on to the $10 \mu \mathrm{m}$ polybeads (through electrostatic interaction) 35 by incubating these vesicles with the beads at $4{ }^{\circ} \mathrm{C}$ overnight under gentle agitation. The fluorophore intensity was analyzed by using BD LSR Fortessa flow cytometry analyser.

Cellular uptake of mCVTs

Fluorophore labelled DOTAP liposomes, CG and mCVTs were produced as described above for the cellular uptake experiment. 3T3-L1 cells with 60-70\% confluency were incubated with fluorophore labelled DOTAP liposomes, CG and mCVTs. The fluorophore intensity was analyzed after 1, 3, 6, 24 and 48 hours of incubation by using BD LSR Fortessa flow cytometry analyser.

\section{Statistical analysis}

All statistical analyses were performed using GraphPad Prism 50 7 using one-way ANOVA, followed by Bonferroni post hoc test. $P<0.05$ was deemed significant.

\section{Conflicts of interest}

The authors declare the following competing financial interest (s): Wei Jiang Goh, Shui Zou and Giorgia Pastorin are the 
inventors of a patent relating to the production and use of CVTs as drug delivery vehicles (PCT/SG2018/050376; Title: Biomolecular Composites Comprising Modified Cell Ghosts).

\section{Acknowledgements}

This work was supported by National University of Singapore (NUS), Department of Pharmacy and by the funding provided by MOE (grant number R-148-000-296-114) and partly supported by the Singapore NMRC (NMRC/OFYIRG/0081/2018 to J. W. W., G. S., B. C. and G. P.). The authors would like to acknowledge A/P Gigi Chiu and A/P Ang Wee Han in NUS for the use of cells and equipment.

\section{References}

1 S. Yamano, J. Dai and A. M. Moursi, Mol. Biotechnol., 2010, 46, 287-300.

2 T. K. Kim and J. H. Eberwine, Anal. Bioanal. Chem., 2010, 397, 3173-3178.

3 J. F. Tan, T. A. Hatton, K. C. Tam and H. P. Too, Biomacromolecules, 2007, 8, 448-454.

4 A. K. Pannier and L. D. Shea, Mol. Ther., 2004, 10, 19-26.

5 T. Niidome and L. Huang, Gene Ther., 2002, 9, 1647-1652.

6 Y. Seow and M. J. Wood, Mol. Ther., 2009, 17, 767-777.

7 G. D. Schmidt-Wolf and I. G. H. Schmidt-Wolf, Trends Mol. Med., 2003, 9, 67-72.

8 E. Jubeli, W. P. D. Goldring and M. D. Pungente, in Methods in Molecular Biology, Humana Press Inc., 2016, vol. 1445, pp. 19-32.

9 P. Midoux, G. Breuzard, J. Gomez and C. Pichon, Curr. Gene Ther., 2008, 8, 335-352.

10 F. Prüfert, S. Bonengel, S. Köllner, J. Griesser, M. D. Wilcox, P. I. Chater, J. P. Pearson and A. Bernkop-Schnürch, Nanomedicine, 2017, 12, 2713-2724.

11 D. Lou and W. M. Saltzman, Nat. Biotechnol., 2000, 18, 3337.

12 S. L. Holmen, M. W. Vanbrocklin, R. R. Eversole, S. R. Stapleton and L. C. Ginsberg, Vitr. Cell. Dev. Biol. Anim. J. Soc. Vitr. Biol., 1995, 31, 347-351.

13 P. Washbourne and A. K. McAllister, Curr. Opin. Neurobiol., $2002,12,566-573$.

14 X. Han, Z. Liu, M. C. Jo, K. Zhang, Y. Li, Z. Zeng, N. Li, Y. Zu and L. Qin, Sci. Adv., 2015, 1, e1500454.

15 G. Kilroy, D. H. Burk and Z. E. Floyd, PLoS One, 2009, 4, e6940.

16 S. M. Tietz and M. Berghoff, J. Biomol. Tech., 2012, 23, 4750.
17 N. Zhao, J. Qi, Z. Zeng, P. Parekh, C. C. Chang, C. H. Tung and Y. Zu, J. Controlled Release, 2012, 159, 104-110.

18 J. Yin, Z. Ma, N. Selliah, D. K. Shivers, R. Q. Cron and T. H. Finkel, J. Immunol. Methods, 2006, 312, 1-11.

19 L. Novo, E. Mastrobattista, C. F. VanNostrum, T. Lammers and W. E. Hennink, Expert Opin. Drug Delivery, 2015, 12, 507512.

20 F. J. Ruiz-Ojeda, J. Plaza-Díaz, M. J. Sáez-Lara and A. Gil, Adv. Nutr., 2019, 10, S31-S48.

21 O. Gresch and L. Altrogge, Methods Mol. Biol., 2012, 801, $65-74$.

22 A. Zotova, E. Lopatukhina, A. Filatov, M. Khaitov and D. Mazurov, Viruses, 2017, 9, 325.

23 H. F. Teixeira, F. Bruxel, M. Fraga, R. S. Schuh, G. K. Zorzi, U. Matte and E. Fattal, Int. J. Pharm., 2017, 534, 356-367.

24 W. J. Goh, C. K. Lee, S. Zou, E. C. Woon, B. Czarny and G. Pastorin, Int. J. Nanomed., 2017, 12, 2759-2767.

25 S. C. Jang, O. Y. Kim, C. M. Yoon, D.-S. Choi, T.-Y. Roh, J. Park, J. Nilsson, J. Lötvall, Y.-K. Kim and Y. S. Gho, ACS Nano, 2013, 7, 7698-7710.

26 W. J. Goh, S. Zou, W. Y. Ong, F. Torta, A. F. Alexandra, R. M. Schiffelers, G. Storm, J.-W. Wang, B. Czarny and G. Pastorin, Sci. Rep., 2017, 7, 14322.

27 L. Kaneti, T. Bronshtein, N. Malkah Dayan, I. Kovregina, N. Letko Khait, Y. Lupu-Haber, M. Fliman, B. W. Schoen, G. Kaneti and M. Machluf, Nano Lett., 2016, 16, 1574-1582.

28 H. M. Byun, D. Suh, H. Yoon, J. M. Kim, H. G. Choi, W. K. Kim, J. J. Ko and Y. K. Oh, Gene Ther., 2004, 11, 492496.

29 T. Li, H. Dong and R. Mo, Nano Res., 2018, 11, 5240-5257.

30 E. V. Batrakova and M. S. Kim, J. Controlled Release, 2015, 219, 396-405.

31 W. J. Goh, S. Zou, B. Czarny and G. Pastorin, Nanoscale, 2018, 10, 6812-6819.

32 W. F. Wolkers, H. T. Oldenhof, F. Tang, J. Han, J. Bigalk and H. Sieme, Langmuir, 2019, 35, 7520-7528.

33 C. Has and P. Sunthar, J. Liposome Res., 2019, 1-30.

34 L. Huang and S. Li, Nat. Biotechnol., 1997, 15, 620-621.

35 M. Agirre, J. Zarate, G. Puras, E. Ojeda and J. Luis Pedraz, Drug Delivery, 2015, 22, 100-110.

36 M. Sancho-Albero, N. Navascués, G. Mendoza, V. Sebastián, M. Arruebo, P. Martín-Duque and J. Santamaría, J. Nanobiotechnol., 2019, 17, 16.

37 I. Hazan-Halevy, D. Rosenblum, S. Weinstein, O. Bairey, P. Raanani and D. Peer, Cancer Lett., 2015, 364, 59-69.

38 A. L. Dordevic, N. Konstantopoulos and D. Cameron-Smith, PLoS One, 2014, 9, e99382.

39 D. E. Murphy, O. G. deJong, M. Brouwer, M. J. Wood, G. Lavieu, R. M. Schiffelers and P. Vader, Exp. Mol. Med., 2019, 51, 1-12. 